\title{
Cloud-Assisted Device Clustering for Lifetime Prolongation in Wireless IoT Networks
}

\author{
Sabin Bhandari, Shree Krishna Sharma, Xianbin Wang \\ Department of Electrical and Computer Engineering \\ Western University, London, Ontario \\ Email: \{sbhanda7, sshar323, xianbin.wang $\} @$ uwo.ca
}

\begin{abstract}
One of the crucial challenges in the recently emerging Internet of Things (IoT) applications is how to handle the massive heterogeneous data generated from a large number of resource-constrained sensors. In this context, cloud computing has emerged as a promising paradigm due to its enormous storage and computing capabilities, thus leading to the IoT-Cloud convergence. In such a framework, IoT devices can be grouped into several clusters and each cluster head can send the aggregated information to the cloud via a gateway for further processing. Although a number of clustering methods have been proposed for the conventional Wireless Sensor Networks (WSNs), it is important to consider specific IoT characteristics while adapting these techniques for wireless IoT networks. One of the important features of IoT networks that can be exploited while developing clustering techniques is the collaborations among heterogeneous IoT devices. In this regard, the network-wide knowledge at the cloud center can be useful to provide information about the device relations to the IoT gateway. Motivated by this, we propose and evaluate a cloud-assisted device interaction-aware clustering scheme for heterogeneous IoT networks. The proposed method considers the joint impact of residual energy and device closeness factor for the effective selection of cluster heads. Our results show that the proposed clustering scheme can significantly prolong the network lifetime, and enhance the overall throughput of a wireless IoT network.
\end{abstract}

Index Terms-Internet of Thing (IoT), Device Interactions, Clustering, Cloud Computing.

\section{INTRODUCTION}

With the rapid advancements in digital electronics and wireless communications, the application areas of Internet of Things (IoT) have increased significantly, and they support a wide range of applications including industrial automation, intelligent transportation, medical, and eHealthcare services [1]. In addition, IoT is also considered as an integral part of future Internet and comprises with the millions of intelligent communicating objects or things. The IoT term is also referred as the Internet of Everything (IoE), and it basically connects the people, things, processes, and data together to fulfill the everyday needs of society in an effective way [2]. The emerging Wireless Sensor Networks (WSNs), Device-toDevice (D2D), and Machine-to-Machine (M2M) technologies have significant impact to extend the sensory capabilities of different sensors, thus enabling the concept of wireless IoT [3]. The widely used Intranet of Things [4] usually refers to local networks with the set of paradigms such as M2M, D2D, and WSN, and only have the regional information. However, IoT networks can exploit comprehensive and historical information by collaborating with different intranets and the cloud server.

The IoT networks usually consist of a large number of sensor nodes, and due to limited network resources, it is inefficient for all nodes to send the sensed data directly to the gateway. Alternatively, the sensor nodes are grouped as a cluster, and each sensor node sends the sensed data to the respective cluster head/aggregator. The aggregator then sends the combined data to the gateway via an Internet link. This approach is more reliable and energy efficient as compared to the flat routing, where each sensor node directly delivers the sensed data to the gateway [5]. Some of the commonly used hierarchical routing or clustering protocols in the conventional WSNs are Low Energy Adaptive Clustering Hierarchy (LEACH), Hybrid Energy Efficient Distributed (HEED), Threshold Sensitive Energy Efficient Sensor Network (TEEN), and Stable Election Protocol (SEP) [6]. The well-known LEACH is one of the pioneer clustering protocols in the field of WSNs, which performs on the basis of distributive clustering model where different sensor nodes are independently responsible to determine their roles on a probabilistic approach and the residual energy. The aforementioned conventional routing protocols are designed based on the energy levels by assuming all sensor nodes are equipped with the same sensing and communicating capabilities [7]. However, these protocols may not be applicable for the next generation IoT networks because of heterogenous features of the employed IoT sensors.

One of the main challenges in IoT networks is to handle the massive amount of heterogeneous data coming from IoT devices/sensors. In this context, cloud computing is considered as an emerging platform to provide the flexible stack of storage, processing capacity, and different software services in a virtualized and scalable manner at reliable costs [8], [9]. Due to the increasing workload, it is very important to utilize the limited resources of wireless IoT networks so that the overall efficiency can be maximized. Recently, Socially Aware Networking (SAN) is emerging as a new paradigm to exploit the social interactions among sensor nodes while devising effective network solutions [10], [11]. Similary, in IoT networks, individual nodes can collaborate with each other to create new services and applications. The properties of Social IoT (SIoT) to improve the specific network performance have been recently presented in [10]. In [11], the authors proposed a cluster formation scheme to categorize the group 
of user equipments by jointly considering the network and social knowledge. However, existing clustering techniques did not consider the joint impact of interactions among the devices and the energy level.

In this paper, a cloud-assisted device interaction-aware clustering scheme is presented for irregularly deployed sensor nodes to enhance the network performance with the objectives of prolonging the network lifetime, maximizing the throughput, and reducing energy consumption. The conventional clustering schemes may help to alleviate some of the above issues, however, they may not be directly applicable for the IoT networks. The interaction profiles of smart devices demand new engineering techniques to make the efficient interconnected network islands. Furthermore, the device closeness factor can measure the strength and bonding between the sensor nodes in their interactions. Therefore, in our proposed scheme, in addition to the commonly used residual energy metric, we incorporate device interaction factor to select the reliable cluster head in a distributed fashion in two-level hierarchical IoT networks. The performance of the proposed scheme is evaluated and compared with the traditional energy level based approach.

The rest of this paper is organized as follows. In Section II, the overall system model of our network is presented. In Section III, the proposed scheme is described in detail. In Section IV, the performance of the proposed scheme is evaluated via computer simulations. Finally, the paper is concluded in Section V.

\section{SYSTEM MODEL}

The industrial IoT is an emerging application of IoT technologies in various scenarios such as automation, intelligence control, smart building, intelligent transportation, and smart grid [12]. The industrial WSN is the key element of the IoT infrastructure to bridge the gap between physical sensors and actuators with the backbone Internet and the cloud. The IoT should provide the reliability and timing predictability to meet the critical requirements of different industrial sub-systems. In this paper, we consider an industrial IoT network, composed of $n$ number of heterogeneous devices deployed in an $l \times l$ rectangular network island (i.e., industrial sub-unit). In each network island, we assume that a fraction of sensor nodes are equipped with more energy resources as compared to the rest of the nodes. Also, these nodes are considered to be more interactive than the remaining ones. These interactive nodes gather more information, and also share the resources and information to the other nodes.

Let $m$ fraction of the total number of sensor nodes have $\alpha$ times more energy and are $\beta$ times more interactive with the neighboring nodes than the normal nodes. We refer the powerful nodes as the advanced nodes and the rest as the normal nodes. In the proposed scheme, all the sensor nodes use the closeness factor, initial energy, and the residual energy level to determine a cluster head. The network is organized as a clustered hierarchial model, in which the cluster-heads are responsible to collect the sensed information from all

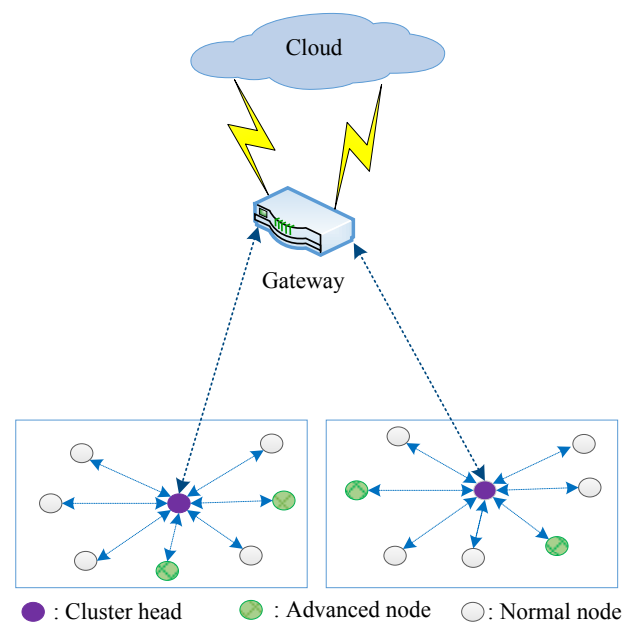

Fig. 1. System model for the proposed clustered hierarchical IoT network.

the nodes within the corresponding clusters and transmit the aggregated information to the gateway as depicted in Fig. 1. In IoT networks, the gateway is responsible for transferring the gathered information to the cloud via wireless Internet links. Moreover, the proposed topology is considered to be static over the time.

The IoT nodes expend various levels of energy depending on their different communication characteristics, interaction behaviors, and short-term link failures. The non-cluster head nodes periodically send this information to the cluster head during the handshaking process, and cluster heads forward this information to the cloud via the gateway. The cloud provides information regarding the election probabilities of normal and advanced nodes to become a cluster head to the cluster heads, and cluster heads subsequently multicast messages during the handshaking process. The number of cluster heads is decided in accordance with the probability that a node becomes a cluster head. In the considered scenario, the cluster heads are chosen probabilistically, and each cluster head broadcasts its information. The sensor nodes receive the number of broadcast messages and measure the signal strength. Then each sensor node sends a join request message to the cluster head based on the measured received signal strength. After the cluster formation, the sensor nodes send the sensed information to the cluster heads in accordance with Time Division Multiple Access (TDMA) fashion. Thereafter, each cluster head aggregates the received information and sends the aggregated data to the cloud via the gateway.

\section{PRoposed Scheme}

In this section, we present the proposed protocol which improves the stability of the hierarchical clustering process by considering different parameters of heterogeneity, i.e., fraction of advanced nodes $m$, closeness factor $\beta$, additional energy factor $\alpha$ between normal and advanced nodes, and residual energy $E_{R}$. The probability of advanced nodes to become a cluster head is higher as compared to the normal nodes, which is also equivalent to a fairness constaint on the interaction among the devices and energy consumption. Let $E_{0}$ be the 
initial energy of each normal sensor node. Then, the energy of each advanced node is given by; $E_{0}(1+\alpha)$. Therefore, the total system energy of the considered heterogeneous setting is given by [13],

$$
n(1-m) E_{0}+n m E_{0}(1+\alpha)=n E_{0}(1+\alpha m) .
$$

The optimal clustering highly depends on the employed energy model. Here, in this paper, we use the radio energy model presented in [14]. In order to achieve an acceptable Signal-to-Noise Ratio (SNR) to transmit the $L$ bit message over $d$ distance, the energy spent by the transmitter is given by,

$$
E_{T x}(L, d)= \begin{cases}L E_{\text {elec }}+L \mathcal{E}_{f s} d^{2}, & \text { if } d<d_{0}, \\ L E_{\text {elec }}+L \mathcal{E}_{m p} d^{4}, & \text { if } d \geq d_{0},\end{cases}
$$

where $E_{\text {elec }}$ is the energy dissipated at the transmitter and receiver to transmit or receive one bit data, which depends on the employed coding, modulation, and filtering techniques. The values of $\mathcal{E}_{f s}$ and $\mathcal{E}_{m p}$ depend on the characteristics of the transmitter amplifier. The distance threshold $d_{0}$, and the energy spent while receiving the $L$ bit message at the receiver is given by [13],

$$
\begin{gathered}
d_{0}=\sqrt{\frac{\mathcal{E}_{f s}}{\mathcal{E}_{m p}}}, \\
E_{R x}=L E_{\text {elec }} .
\end{gathered}
$$

By assuming the area equal to an $l \times l$ square meters and $n$ number of nodes are uniformly distributed, the average distances between the cluster head to the gateway $d_{2 G}$, and IoT nodes to the cluster head $d_{2 C H}$ are given by,

$$
\begin{aligned}
& d_{2 G}=0.765 \frac{l}{2}, \\
& d_{2 C H}=\frac{l}{\sqrt{2 k \pi}},
\end{aligned}
$$

where $k$ is the number of clusters.

Also, the optimal number of constructed clusters $k_{o p t}$ and the optimal probability of a node to become a cluster head $P_{\text {opt }}$ can be computed as follows [13],

$$
\begin{gathered}
k_{o p t}=\sqrt{\frac{n}{2 \pi}} \frac{l}{d_{2 G}}, \\
P_{o p t}=\frac{k_{o p t}}{n} .
\end{gathered}
$$

The interactions among IoT nodes have a great impact on seamless communication. The closeness factor gives the strength among the sensor nodes and thus reflects the strong or weak bonding between two nodes. We represent the interactions among $n$ nodes by a symmetric matrix $S_{n}=\left\{s_{i, j}\right\}_{n \times n}$, where the matrix element $s_{i, j}$ gives the degree of closeness between $i^{\text {th }}$ and $j^{\text {th }}$ sensor nodes, $\forall i, j \in n$. We define the range of $s_{i, j}$ as $s_{i, j} \in[0,1]$, and assume the bondness between two nodes is directly proportional to $s_{i, j}$. The overall closeness between the $i^{t h}$ sensor node and other nodes is given by,

$$
S_{W}=\sum_{i, j \in n} s_{i, j}
$$

In our proposed scheme, we derive cluster head election probabilities by assigning weights to the optimal probability given by (8). The weighting values are based on the energy and the closeness factor. Let $P_{n r m}$ and $P_{a d v}$ are the weighted election probabilities for normal and advanced nodes respectively and calculated by,

$$
\begin{gathered}
P_{n r m}=\frac{P_{o p t}}{1+(\alpha+\beta) m}, \\
P_{a d v}=\frac{P_{o p t}}{1+(\alpha+\beta) m}(1+\alpha+\beta) .
\end{gathered}
$$

Let $\hat{E}_{R}$ and $\hat{S}_{W}$ be the normalized residual energy and normalized closeness of the corresponding node. Then, the threshold values to become a cluster head for normal and advanced nodes are respectively given by,

$$
\begin{gathered}
P_{T}\left(n_{n r m}\right)=\left\{\begin{array}{cc}
\frac{P_{n r m}}{1-P_{n r m}\left(r . m o d\left(\frac{1}{P_{n r m}}\right)\right)} \hat{E}_{R n r m} & \hat{S}_{W n r m} \\
0 & \text { if } n_{n r m} \in G^{\prime}
\end{array}\right. \\
P_{T}\left(n_{a d v}\right)=\left\{\begin{array}{cc}
\frac{P_{a d v}}{1-P_{a d v}\left(r . m o d\left(\frac{1}{P_{a d v}}\right)\right)} \hat{E}_{R a d v} \hat{S}_{W a d v} \\
\text { if } n_{a d v} \in G^{\prime \prime}
\end{array}\right.
\end{gathered}
$$

where $r$ is the current round, $G^{\prime}$ and $G^{\prime \prime}$ are the set of nodes that have not become the cluster heads in the last $\frac{1}{P_{n r m}}$ and $\frac{1}{P_{a d v}}$ rounds of epoch, respectively. The average number of cluster heads per round $N_{C H}$ is given by,

$$
N_{C H}=n(1-m) P_{n r m}+n m P_{a d v} .
$$

By substituting the values of $P_{n r m}$ and $P_{a d v}$ from (10) and (11) in (14), we obtain following,

$$
N_{C H}=n P_{o p t} \text {. }
$$

\section{PERformance AnAlysis}

In this section, we evaluate and analyze the performance of the proposed scheme using MATLAB. We simulate an IoT network with $n=100$ nodes uniformly distributed in a field of area $100 \mathrm{~m} \times 100 \mathrm{~m}$. The gateway is positioned at the center of the sensing area and the maximum distance of any node from the gateway is considered to be $70 \mathrm{~m}$. The simulation parameters are listed in Table I [13]. For the considered value of $P_{o p t}=0.1$, we obtained different probabilities for each type of nodes to become a cluster head with the values of $\alpha$, $\beta$, and $m$ by using (10) and (11) respectively. We performed simulations for different values of $\beta$ by keeping $\alpha=2$

TABLE I

SimUlation PARAMENTERS

\begin{tabular}{cc}
\hline Parameters & Value \\
\hline$E_{\text {elec }}$ & $50 \mathrm{~nJ} / \mathrm{bit}$ \\
$\mathcal{E}_{\text {fs }}$ & $10 \mathrm{pJ} / \mathrm{bit} / \mathrm{m}^{2}$ \\
$\mathcal{E}_{m p}$ & $0.0013 \mathrm{pJ} / \mathrm{bit} / \mathrm{m}^{4}$ \\
$E_{0}$ & $0.5 \mathrm{~J}$ \\
$d_{0}$ & $70 \mathrm{~m}$ \\
Message size & $4000 \mathrm{bits}$ \\
$P_{\text {opt }}$ & 0.1 \\
\hline
\end{tabular}




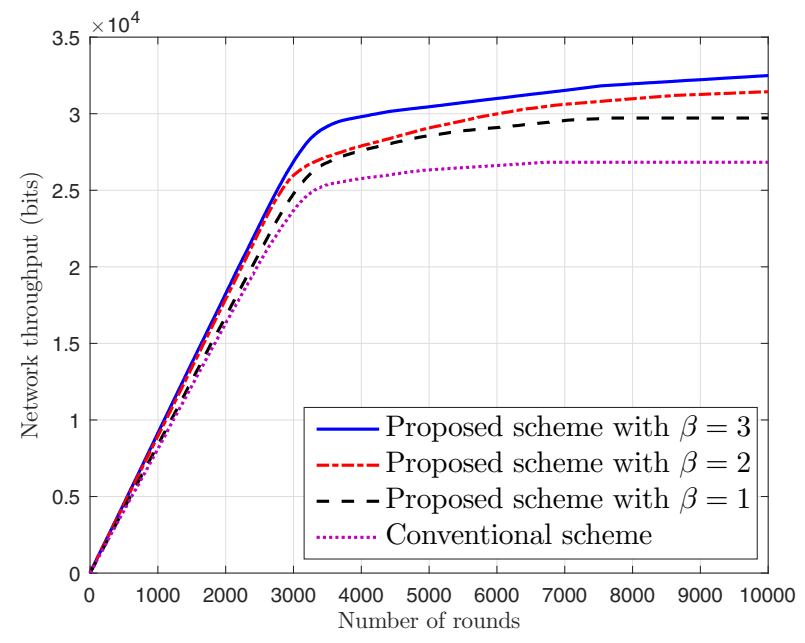

Fig. 2. Performance comparison of the proposed approach with the conventional in terms of network throughput.

and $m=0.1$. However, for the simulations of conventional scheme, we considered $\beta=0$ and $\hat{S}_{W}=1$.

Figure 2 shows that the throughput of the network, i.e., data sent from the cluster heads to the gateway. As we increase the closeness factor of advanced nodes from $\beta=1$ to 3 , the throughput also increases because of more interaction and higher energy level of advanced nodes as compared to the normal nodes. From the presented results in Fig. 2, we can note that the proposed scheme outperforms the conventional one in terms of the network throughput.

Figure 3 depicts the number of alive nodes per round. It is clearly observed that the number of alive nodes per round increases with $\beta$ within its considered range. In this way, the network life time is remarkably prolonged. From the presented results, it can be deduced that the proposed scheme successfully extends the network stability (i.e., alive nodes) by being aware of the network heterogeneity through assigning the probabilities of cluster head election by weighted closeness factor and the residual energy.

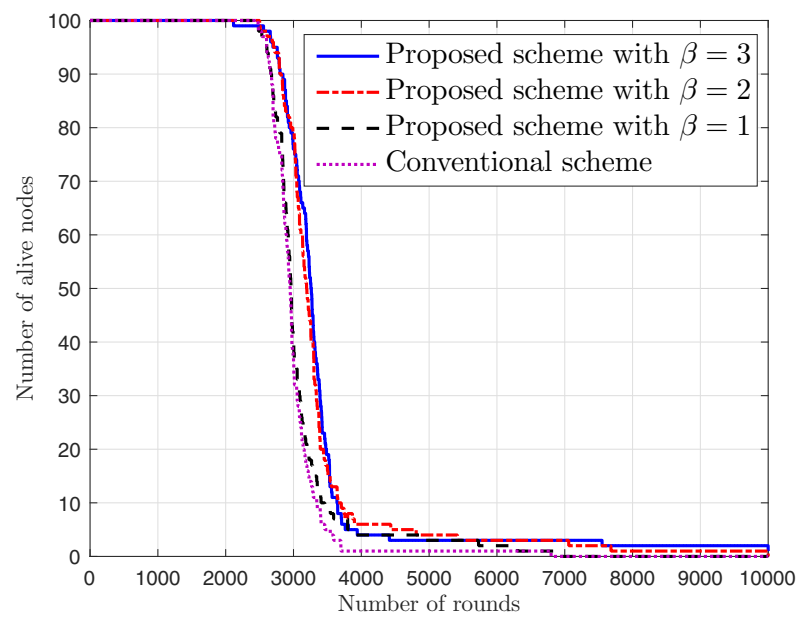

Fig. 3. Performance comparison of the proposed approach with the conventional in terms of network lifetime.

\section{CONClusion}

The IoT nodes are heterogeneous in terms of their processing capabilities, energy, and operating bandwidth. They can help each other by sharing their status as well as the contextual information, and these interactions have a great impact in the design of efficient and reliable wireless IoT networks. In this paper, we proposed a cloud-assisted clustering scheme where each sensor node elects itself as a cluster head on the basis of initial energy, residual energy, and the closeness factor. In the proposed framework, the cloud facilitates the clustering process by providing the election probabilities of normal and advanced nodes to the current cluster heads according to the energy and device closeness factors. We analyzed the impact of heterogeneity of IoT networks in terms of energy and bondness among IoT nodes. With the help of numerical results, it has been demonstrated that the proposed scheme provides longer network stability and higher throughput as compared to the conventional one. In our future work, we plan to extend the proposed scheme for other emerging networks such as Internet of Vehicle (IoV) network, and to develop novel data aggregation and prioritization algorithms for wireless IoT.

\section{REFERENCES}

[1] A. Al-Fuqaha, M. Guizani, M. Mohammadi, M. Aledhari, and M. Ayyash, "Internet of things: A survey on enabling technologies, protocols, and applications," IEEE Commun. Surveys Tuts., vol. 17, no. 4, pp. 2347-2376, Fourthquarter 2015.

[2] S. K. Sharma, T. E. Bogale, S. Chatzinotas, X. Wang, and L. B. Le, "Physical layer aspects of wireless IoT," in Proc. IEEE ISWCS, Sept 2016, pp. 304-308.

[3] J. Gubbi, R. Buyya, S. Marusic, and M. Palaniswami, "Internet of things (IoT): A vision, architectural elements, and future directions," Future Gener. Comput. Syst., vol. 29, no. 7, pp. 1645-1660, 2013.

[4] M. Zorzi, A. Gluhak, S. Lange, and A. Bassi, "From today's intranet of things to a future internet of things: a wireless- and mobility-related view," IEEE Wireless Commun., vol. 17, no. 6, pp. 44-51, Dec 2010.

[5] H. Shin, S. Moh, I. Chung, and M. Kang, "Equal-size clustering for irregularly deployed wireless sensor networks," Wireless Pers. Commun., vol. 82, no. 2, pp. 995-1012, 2015.

[6] S. P. Singh and S. Sharma, "A survey on cluster based routing protocols in wireless sensor networks," Procedia Comput. Sci., vol. 45, pp. 687695, 2015.

[7] A. Bagula, A. P. Abidoye, and G.-A. L. Zodi, "Service-aware clustering: An energy-efficient model for the internet-of-things," Sensors, vol. 16, no. 1 , p. $9,2015$.

[8] M. M. Hassan, H. S. Albakr, and H. Al-Dossari, "A cloud-assisted internet of things framework for pervasive healthcare in smart city environment," in Proc. of EMASC. ACM, 2014, pp. 9-13.

[9] S. K. Sharma and X. Wang, "Live data analytics with collaborative edge and cloud processing in wireless IoT networks," IEEE Access, vol. PP, no. 99, pp. 1-1, 2017.

[10] A. M. Ortiz, D. Hussein, S. Park, S. N. Han, and N. Crespi, "The cluster between internet of things and social networks: Review and research challenges," IEEE Internet Things J., vol. 1, no. 3, pp. 206-215, June 2014.

[11] G. Zhang, K. Yang, and H. H. Chen, "Socially aware cluster formation and radio resource allocation in D2D networks," IEEE Wireless Commun., vol. 23, no. 4, pp. 68-73, Aug 2016.

[12] L. D. Xu, W. He, and S. Li, "Internet of things in industries: A survey," IEEE Trans. Ind. Informat, vol. 10, no. 4, pp. 2233-2243, Nov 2014.

[13] G. Smaragdakis, I. Matta, and A. Bestavros, "SEP: A stable election protocol for clustered heterogeneous wireless sensor networks," Boston University Computer Science Department, Tech. Rep., 2004.

[14] W. Heinzelman, A. Chandrakasan, and H. Balakrishnan, "An application-specific protocol architecture for wireless microsensor networks," IEEE Trans. Wireless Commun., vol. 1, no. 4, pp. 660-670, Oct 2002. 